\title{
СТАТИСТИКА ЭСТОНСКОЙ УСТНОЙ РЕЧИ
}

\author{
(Представлена Н. Алумяэ)
}

Как известно, устная речь состоит из последовательности звуков, которые произносятся, как правило, слитно, с паузами только после отдельных слов или групп звуков. По объему фраз, т. е. по количеству слов во фразе, устная речь отличается от письменной $\left[{ }^{1}\right]$. Каждый звук имеет индивидуальный характер и оказывает в процессе речи некоторое влияние на конечное состояние предыдущего и начальное состояние следующего за ним звука. Очень часто в устной речи окончания слов произносятся с фонетическими ошибками либо не произносятся совсем, нередко в речь вкрадываются слова, лишенные какого-либо смысла. В данной работе излагаются статистические данные о частоте появления в речи отдельных звуков, а также двух- и трехзвуковых сочетаний и сравниваются в этом плане устная и письменная формы эстонского языка.

\section{Анализируемый материал}

Произвольно выбранные отрезки речи между двумя или более лицами, услышанные на собраниях, в магазинах, по радио или телевидению, были записаны на' магнитную ленту, переведены на язык фонетических символов, перфорированы и проанализированы на ЭВМ. Анализ был проведен по синтагмам, т. е. по паузам в слитной речи. Выборка содержала 105942 фонетических символа (звукотипа), образующих в совокупности 19620 слов и 4923 синтагмы.

Чтобы в тексте отличать звукотипы от букв, первые заключены в скобки, а вторые выделены курсивом.

В алфавите эстонского языка 23 буквы. При написании иностранных имен и выражений используются еще $c, \check{c}, f, q, \check{s}, z, \check{z}, w, x, y$, из них нанболее часто встречается $f$. Звукотипов в нашем материале больше их насчитывается 32 (см. табл. 1). Это значит, что на письме разные звукотипы обозначаются одними и теми же буквами. В эстонском языке согласные $l, t, n, s, d$ могут быть как па:гатализованными, так и непалатализованными. Первые отмечены нами апострофом. Наиболее часто палатализуются $l^{\prime}$ и $t^{\prime}-31,1$ и $29,7 \%$ соответственно, реже $n^{\prime}$ и $s^{\prime}-$ 19,7 и $16,4 \%$ соответственно и редко $d^{\prime}-3,3 \%$. В «Oigekeelsussõnaraamat» («Орфографический словарь», Таллин, «Валгус», 1976), содержащем более 100000 слов, есть 61 пара таких слов, которые пишутся одинаково, но вследствие палатализации имеют различные значения. В тех случаях, когда /u/ в комбинациях /au/ и / ue/ произносится как нечто среднее между /u/ и /v/, он выделен нами в самостоятельный звукотип и отмечен как $/ \mathrm{w} /$. 
Таблича $I$

\begin{tabular}{|c|c|c|c|c|c|}
\hline \multirow[b]{2}{*}{ Звук } & \multirow{2}{*}{\multicolumn{2}{|c|}{ Характерное слово }} & \multicolumn{2}{|c|}{ Частотность } & \multirow{2}{*}{$\begin{array}{c}\text { Доверительные } \\
\text { границы при } 99 \% \text {-ной } \\
\text { вероятности }\end{array}$} \\
\hline & & & абсолют. & относит., \% & \\
\hline$|\mathrm{a}|$ & kana & курица & 12304 & 11.61 & $11,36-11,87$ \\
\hline$|\mathrm{e}|$ & neli & четыре & 12215 & 11,53 & $11,28-11,78$ \\
\hline$|\mathrm{i}|$ & pime & темно & 10465 & 9,88 & $9,65-10,12$ \\
\hline$|t|$ & ketas & диск & 8204 & 7,75 & $7,53-7,97$ \\
\hline $\mid \mathrm{s} /$ & võsa & поросль & 6731 & 6,35 & $6,15-6,55$ \\
\hline $11 /$ & väle & прыткий & 5777 & 5,45 & $5,27-5,63$ \\
\hline $\mid \mathrm{u} /$ & tuli & огонь & 5446 & 5,14 & $4,97-5,32$ \\
\hline $\ln /$ & pinu & поленница & 5425 & 5,12 & $4,95-5,30$ \\
\hline$|\mathrm{k}|$ & lake & пойло & 5056 & 4,77 & $4,60-4,94$ \\
\hline dd/ & ida & восток & 4202 & 3,97 & $3,82-4,13$ \\
\hline$/ \mathrm{m} /$ & lame & плоский & 4193 & 3,96 & $3,81-4,12$ \\
\hline$|\mathrm{o}|$ & $\log i$ & лаг & 3786 & 3,58 & $3,44-3,73$ \\
\hline$|z|$ & tagasi & назад & 2937 & 2,77 & $2,64-2,90$ \\
\hline$|r|$ & tara & забор & 2527 & 2,39 & $2,27-2,51$ \\
\hline$|\mathrm{j}|$ & maja & дом & 2422 & 2,29 & $2,17-2,41$ \\
\hline$|\mathrm{v}|$ & kava & план & 2397 & 2,26 & $2,14-2,38$ \\
\hline |gl & nuga & нож & 2295 & 2,17 & $2,06-2,29$ \\
\hline$|\mathrm{p}|$ & hapu & кислый & 1732 & 1,64 & $1,54-1,74$ \\
\hline /h/ & rahe & град & 1604 & 1,51 & $1,42-1,61$ \\
\hline |ä| & nägu & лицо & 1505 & 1,42 & $1,33-1,52$ \\
\hline$|\overline{0}|$ & kõle & нежилой & 1460 & 1,38 & $1,29-1,48$ \\
\hline $\mid \mathrm{b} /$ & tuba & комната & 1085 & 1,02 & $0,942-1,10$ \\
\hline /ü/ & püha & святой & 956 & 0,902 & $0,827-0,977$ \\
\hline$\left|1^{\prime}\right|$ & kull & ястреб & 306 & 0,288 & $0,241-0,336$ \\
\hline$|\ddot{\mid}|$ & lōga & жижа & 230 & 0,217 & $0,180-0,254$ \\
\hline$|\eta|$ & pang & ведро & 171 & 0,161 & $0,130-0,193$ \\
\hline$\left|s^{\prime}\right|$ & kass & кошка & 142 & 0,134 & $0,105-0,163$ \\
\hline$\left|\mathrm{n}^{\prime}\right|$ & kann & игрушка & 132 & 0,124 & $0,097-0,152$ \\
\hline $\mid \mathrm{f} /$ & faas & фаза & 104 & 0,098 & $0,073-0,123$ \\
\hline$|w|$ & kaua, uuesti & долго, снова & 69 & 0,065 & $0,044-0,085$ \\
\hline$\left[\mathrm{t}^{\prime} /\right.$ & kott & мешок & 41 & 0,039 & $0,023-0,054$ \\
\hline \multirow[t]{2}{*}{$\left|\mathrm{d}^{\prime}\right|$} & lodi & барка & 13 & 0,012 & $0,004-0,021$ \\
\hline & & Всего & 105942 & 10 & \\
\hline
\end{tabular}

Таблица 2

\section{Гласные}

передние делабиализованные /i, e, ä/

\section{Согласные}

глухие взрывные /p, t, t', k/

глухие фрикативные /f, s, s', h/

полузвонкие взрывные /b, d, d', g/

звонкие фрикативные $/ \mathrm{v}, \mathrm{z}, \mathrm{w} /$

носовые $/ \mathrm{m}, \mathrm{n}, \mathrm{n}^{\prime}, \eta /$

латеральные $/ 1, \mathrm{l}^{\prime} /$

дрожащий $/ \mathrm{r}$ /

полугласный /j/ 


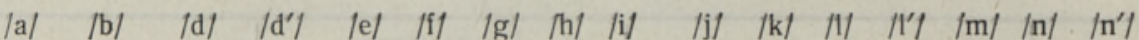
$\begin{array}{llllllllllllllll}\text { la/ } & 341 & 692 & 3 & 366 & 13 & 572 & 482 & 351 & 364 & 832 & 1082 & 127 & 699 & 756 & 20\end{array}$ $\begin{array}{llllllllllllllll}\mid \mathrm{b} / & 177 & 1 & 0 & 113 & 1 & 1 & 0 & 115 & 16 & 56 & 40 & 0 & 34 & 32 & 0\end{array}$ /d/ $\quad \begin{array}{lllllllllllllll}1102 & 0 & 0 & 485 & 2 & 2 & 0 & 330 & 50 & 168 & 120 & 0 & 137 & 115 & 0\end{array}$ $\begin{array}{lrrrlllrrrrrrrrrrr}\mid \mathrm{d}^{\prime} / & 0 & 0 & 0 & & 0 & 0 & 0 & 0 & 13 & 0 & 0 & 0 & 0 & 0 & 0 & 0 \\ \mid \mathrm{e} / & 693 & 157 & 630 & 0 & & 19 & 504 & 167 & 1101 & 141 & 791 & 1504 & 43 & 708 & 754 & 65\end{array}$ |f/ $\quad \begin{array}{lllllllllllllll} & 7 & 0 & 0 & 0 & 12 & 0 & 0 & 38 & 0 & 0 & 1 & 0 & 0 & 0\end{array}$ $\begin{array}{llllllllllllllll}\text { lg/ } & 717 & 0 & 1 & 0 & 247 & 0 & 0 & 553 & 3 & 6 & 11 & 0 & 12 & 16 & 0\end{array}$ /h/ $\quad \begin{array}{lllllllllllllll}242 & 0 & 1 & 0 & 456 & 0 & 0 & 74 & 32 & 89 & 1 & 0 & 14 & 22 & 0\end{array}$ (i) $\quad \begin{array}{lllllllllllllll}134 & 154 & 1007 & 0 & 128 & 6 & 256 & 96 & 318 & 937 & 599 & 3 & 666 & 1159 & 6\end{array}$

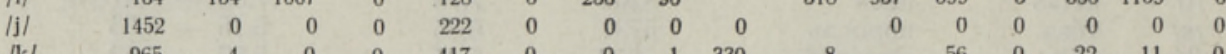

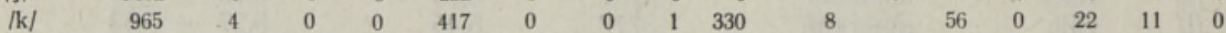
lil $\quad \begin{array}{llllllllllllllll}535 & 9 & 177 & 0 & 1761 & 2 & 87 & 6 & 986 & 52 & 110 & 0 & 208 & 103 & 0\end{array}$ $\begin{array}{lrrrrrrrrrrrrrrrr}\left|1^{\prime}\right| & 1 & 3 & 8 & 0 & 2 & 0 & 13 & 0 & 20 & 210 & 5 & 1 & & 21 & 1 & 0 \\ \mid \mathrm{m} / & 1122 & 61 & 0 & 0 & 946 & 1 & 13 & 1 & 1135 & 7 & 18 & 12 & 0 & & 63 & 0\end{array}$ $\begin{array}{lrrrrrrrrrrrrrrrr}\mid \mathrm{n} / & 693 & 2 & 602 & 0 & 963 & 15 & 63 & 0 & 808 & 54 & 150 & 31 & 0 & 95 & & 0 \\ \left\lfloor\mathrm{n}^{\prime} \mid\right. & 0 & 0 & 57 & 0 & 0 & 1 & 0 & 0 & 40 & 0 & 0 & 0 & 0 & 0 & 0 & \end{array}$

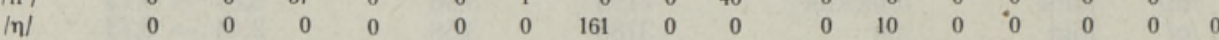
$\begin{array}{lllllllllllllllll}|\mathrm{o}| & 13 & 37 & 99 & 10 & 36 & 3 & 95 & 282 & 63 & 32 & 76 & 937 & 5 & 232 & 1097 & 6\end{array}$

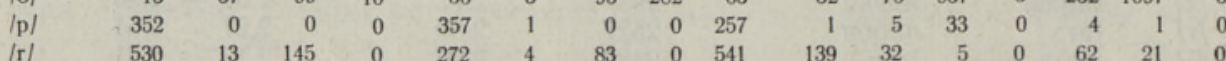

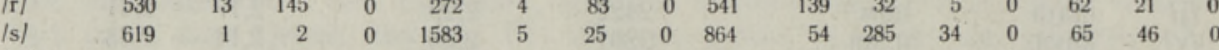
$\begin{array}{lllllllllllllllll}\left|\mathbf{s}^{\prime}\right| & 1 & 0 & 0 & 0 & 1 & 0 & 0 & 0 & 32 & 70 & 16 & 1 & 0 & 0 & 1 & 0\end{array}$ $\begin{array}{lllllllllllllllll}|z| & 152 & 2 & 0 & 0 & 936 & 10 & 6 & 0 & 501 & 27 & 98 & 44 & 0 & 93 & 66 & 0\end{array}$ $\begin{array}{lrrrrrrrrrrrrrrrr}|t| & 1455 & 3 & 0 & 0 & 1602 & 10 & 0 & 1 & 535 & 69 & 238 & 236 & 0 & 186 & 136 & 1 \\ \left|t^{\prime}\right| & 0 & 0 & 0 & 0 & 1 & 0 & 0 & 0 & 9 & 0 & 4 & 0 & 0 & 1 & 0 & 0\end{array}$ $\begin{array}{lrrrrrrrrrrrrrrrr}|\mathrm{u}| & 73 & 199 & 618 & 0 & 35 & 6 & 278 & 136 & 406 & 61 & 206 & 612 & 11 & 199 & 332 & 34 \\ \mid \mathrm{v} / & 887 & 0 & 0 & 0 & 195 & 1 & 0 & 0 & 301 & 4 & 11 & 5 & 0 & 2 & 2 & 0\end{array}$

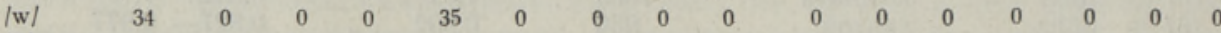
$\begin{array}{lllllllllllllllll}|\overline{0}| & 0 & 10 & 7 & 0 & 46 & 0 & 0 & 82 & 667 & 11 & 3 & 43 & 1 & 20 & 104 & 0\end{array}$ $\begin{array}{lllllllllllllllll}\text { la/ } & 0 & 72 & 12 & 0 & 113 & 0 & 122 & 208 & 306 & 0 & 57 & 63 & 88 & 2 & 49 & 0\end{array}$ /ü/ $\quad$\begin{tabular}{rrrrrrrrrrrrrrrrr}
1 & 7 & 109 & 0 & 0 & 0 & 8 & 138 & 4 & 6 & 110 & 201 & 27 & 155 & 9 & 0 \\
\hline
\end{tabular}

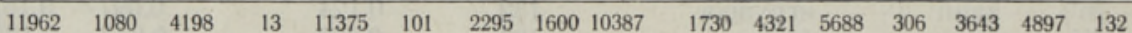

\begin{tabular}{|c|c|c|c|c|c|c|c|c|c|c|c|c|c|c|c|c|}
\hline$|\mathrm{a}|$ & /b/ & /d/ & $\left|\mathrm{d}^{\prime}\right|$ & $|e|$ & /f/ & $|\mathrm{g}|$ & /h/ & /i/ & $\mid \mathrm{j} /$ & $/ \mathrm{k} /$ & AI & $/ I^{\prime} \mid$ & $/ \mathrm{m} /$ & $|\mathrm{n}|$ & $\left|\mathrm{n}^{\prime}\right|$ & $\mid \eta /$ \\
\hline & 166 & 906 & $\theta$ & 666 & 7 & 596 & 191 & 133 & 1293 & 920 & 508 & 1 & 996 & 651 & 0 & 0 \\
\hline 285 & & 0 & 0 & 134 & 0 & 0 & 0 & 140 & 0 & 4 & 9 & 3 & 61 & 2 & 0 & 0 \\
\hline 595 & 1 & & 0 & 524 & & 1 & 0 & 886 & 0 & 0 & 176 & 0 & 0 & 582 & 56 & 0 \\
\hline 3 & 0 & 0 & & 0 & 0 & 0 & 0 & 0 & 0 & 0 & 0 & 0 & 0 & 0 & 0 & 0 \\
\hline 364 & 108 & 446 & 0 & & 12 & 233 & 427 & 128 & 219 & 406 & 1574 & 2 & 903 & 874 & 0 & 0 \\
\hline 13 & 1 & 2 & 0 & 19 & & 0 & 0 & 6 & 0 & 0 & 2 & 0 & 1 & 15 & 0 & 0 \\
\hline 572 & 1 & 2 & 0 & 495 & 0 & & 0 & 256 & 0 & 0 & 87 & 13 & 58 & 0 & 161 & 95 \\
\hline 333 & 0 & 0 & 0 & 165 & 0 & 0 & & 96 & 0 & 1 & 6 & 0 & 1 & 0 & 0 & 0 \\
\hline 346 & 98 & 286 & 10 & 1075 & 38 & 453 & 73 & & 0 & 305 & 933 & 20 & 1124 & 674 & 35 & 0 \\
\hline 364 & 16 & 50 & 0 & 141 & 0 & 3 & 32 & 318 & & 8 & 52 & 210 & 7 & 54 & 1 & 0 \\
\hline 830 & 56 & 168 & $\theta$ & 787 & $\theta$ & 6 & 89 & 901 & $\theta$ & & $11 \theta$ & 5 & 18 & 150 & $\theta$ & $1 \theta$ \\
\hline 1023 & 40 & 120 & 0 & 1425 & 1 & 11 & 1 & 573 & 0 & 56 & & 1. & 12 & 31 & 0 & 0 \\
\hline 127 & 0 & 0 & 0 & 43 & 0 & 0 & 0 & 3 & 0 & 0 & 0 & & 0 & 0 & 0 & 0 \\
\hline 689 & 34 & 137 & 0 & 680 & 0 & 12 & 14 & 666 & 0 & 22 & 201 & 21 & $c^{2}$ & 95 & 0 & 0 \\
\hline 725 & 32 & 115 & 0 & 741 & 0 & 16 & 22 & 1108 & 0 & 11 & 103 & 1 & 63 & & 0 & 0 \\
\hline 20 & 0 & 0 & 0 & 63 & 0 & 0 & 0 & 6 & 0 & 0 & 0 & 0 & 0 & 0 & & 0 \\
\hline 17 & 0 & 0 & 0 & 1 & 0 & 0 & 0 & 112 & 0 & 0 & 0 & 0 & 0 & 0 & 0 & \\
\hline 253 & 83 & 155 & 0 & 291 & 29 & 10 & 11 & 354 & 40 & 775 & 230 & 1 & 104 & 287 & 0 & 0 \\
\hline 287 & 22 & 85 & 0 & 284 & 1 & 3 & 2 & 163 & 0 & 9 & 53 & 0 & 27 & 55 & 0 & 0 \\
\hline 465 & 27 & 37 & 0 & 405 & 0 & 36 & 0 & 139 & 0 & 42 & 11 & 0 & 1 & 17 & 0 & 0 \\
\hline 982 & 74 & 176 & 0 & 876 & 0 & 15 & 14 & 1178 & 0 & 714 & 71 & 1 & 19 & 230 & 10 & 0 \\
\hline 113 & 0 & 0 & 0 & 4 & 0 & 0 & 0 & 4 & 0 & 0 & 0 & 0 & 0 & 1 & 0 & 0 \\
\hline 443 & 0 & 6 & 0 & 384 & 0 & 0 & 0 & 989 & 0 & 0 & 24 & 0 & 8 & 6 & 0 & 0 \\
\hline 1126 & 39 & 112 & 0 & 1139 & 0 & 4 & 328 & 848 & 0 & 487 & 15 & 15 & 20 & 199 & 18 & 0 \\
\hline 5 & 0 & 0 & 0 & 5 & 0 & 0 & 0 & 0 & 0 & 0 & 0 & 0 & 0 & 0 & 0 & 0 \\
\hline 150 & 26 & 340 & 0 & 26 & 4 & 518 & 59 & 30 & 476 & 816 & 232 & 1 & 290 & 406 & 0 & 0 \\
\hline 423 & 43 & 132 & 0 & 425 & 0 & 4 & 54 & 251 & 0 & 21 & 57 & 1 & 20 & 112 & 0 & 0 \\
\hline 0 & 0 & 0 & 0 & 1 & 0 & 0 & 0 & 0 & 0 & 0 & 0 & 0 & 0 & 1 & 0 & 0 \\
\hline 33 & 4 & 15 & 0 & 39 & 0 & 1 & 0 & 26 & 41 & 185 & 49 & 0 & 137 & 53 & 0 & 0 \\
\hline 14 & 37 & 14 & 0 & 20 & 1 & 0 & 0 & 16 & 154 & 110 & 151 & 0 & 80 & 182 & 0 & 0 \\
\hline 8 & 3 & 0 & 0 & 5 & 1 & 0 & 0 & 20 & 0 & 1 & 5 & 0 & 12 & 6 & 0 & 0 \\
\hline 91 & 18 & 34 & 0 & 49 & 10 & 102 & 0 & 36 & 1 & 181 & 43 & 1 & 19 & 122 & & \\
\hline
\end{tabular}




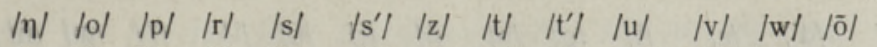

Таблица 3

\begin{tabular}{|c|c|c|c|c|c|c|c|c|c|c|c|c|c|c|c|c|}
\hline 17 & 253 & 287 & 470 & 990 & 113 & 524 & 1156 & 7 & 150 & 433 & 0 & 33 & 14 & 8 & 91 & 11246 \\
\hline 0 & 83 & 22 & 27 & 74 & 0 & 0 & 39 & 0 & 26 & 43 & 0 & 4 & 37 & 3 & 18 & 962 \\
\hline 0 & 155 & 85 & 37 & 176 & 0 & 6 & 112 & 0 & 347 & 132 & 0 & 15 & 14 & 0 & 34 & 3624 \\
\hline 0 & 0 & 0 & 0 & 0 & 0 & 0 & 0 & 0 & 0 & 0 & 0 & 0 & 0 & 0 & 0 & 13 \\
\hline 1 & 291 & 284 & 427 & 895 & 5 & 475 & 1167 & 7 & 26 & 430 & 1 & 39 & 20 & 5 & 49 & 11399 \\
\hline 0 & 29 & 1 & 0 & 0 & 0 & 0 & 0 & 0 & 4 & 0 & 0 & 0 & 1 & 1 & 10 & 104 \\
\hline 0 & 10 & 3 & 36 & 15 & 0 & 0 & 4 & 0 & 540 & 4 & 0 & 1 & 0 & 0 & 102 & 2281 \\
\hline 0 & 12 & 2 & 0 & 14 & 0 & 0 & 339 & 0 & 61 & 54 & 0 & 0 & 0 & 0 & 0 & 1413 \\
\hline 112 & 355 & 164 & 139 & 1209 & 4 & 1070 & 872 & 0 & 30 & 253 & 0 & 26 & 16 & 20 & 36 & 9775 \\
\hline 0 & 41 & 0 & 0 & 0 & 0 & 0 & 0 & 0 & 510 & 0 & 0 & 41 & 154 & 0 & 1 & 2421 \\
\hline 0 & 775 & 9 & 42 & 804 & 0 & 0 & 96 & 0 & 834 & 21 & 0 & 185 & 110 & 1 & 181 & 4872 \\
\hline 0 & 231 & 53 & 11 & 71 & 0 & 24 & 562 & 0 & 237 & 57 & 0 & 49 & 151 & 5 & 43 & 5530 \\
\hline 0 & 1 & 0 & 0 & 1 & 0 & 0 & 15 & 0 & 1 & 1 & 0 & 0 & 0 & 0 & 1 & 305 \\
\hline 0 & 104 & 27 & 1 & 19 & 0 & 8 & 20 & 0 & 294 & 20 & 0 & 137 & 80 & 12 & 19 & 4120 \\
\hline 0 & 295 & 55 & 17 & 230 & 1 & 6 & 200 & 0 & 411 & 112 & 0 & 53 & 182 & 6 & 122 & 5166 \\
\hline 0 & 0 & 0 & 0 & 10 & 0 & 0 & 22 & 0 & 0 & 0 & 0 & 0 & 0 & 0 & 0 & 130 \\
\hline & 0 & 0 & 0 & 0 & 0 & 0 & 0 & 0 & 0 & 0 & 0 & 0 & 0 & 0 & 0 & 171 \\
\hline 10 & & 46 & 326 & 98 & 1 & 85 & 123 & 17 & 6 & 39 & 0 & 1 & 2 & 0 & 3 & 3780 \\
\hline 0 & 149 & & 117 & 34 & 0 & 0 & 15 & 0 & 151 & 1 & 0 & 75 & 142 & 10 & 13 & 1718 \\
\hline 0 & 154 & 10 & & 33 & 0 & 7 & 123 & 0 & 115 & 128 & 0 & 13 & 46 & 0 & 16 & 2492 \\
\hline 0 & 183 & 152 & 7 & & 0 & 0 & 1763 & 0 & 397 & 98 & 0 & 111 & 4 & 9 & 50 & 6357 \\
\hline 0 & 1 & 0 & 0 & 0 & & 0 & 16 & 0 & 0 & 1 & 0 & 0 & 0 & 0 & 1 & 141 \\
\hline 0 & 146 & 50 & 23 & 137 & 0 & & 78 & 0 & 133 & 73 & 0 & 7 & 11 & 1 & 10 & 2604 \\
\hline 0 & 282 & 95 & 177 & 609 & 0 & 0 & & 0 & 1010 & 126 & 0 & 80 & 216 & 144 & 70 & 7281 \\
\hline 0 & 1 & 0 & 0 & 17 & 0 & 0 & 1 & & 0 & 2 & 0 & 0 & 0 & 0 & 1 & 37 \\
\hline 5 & 44 & 76 & 255 & 414 & 14 & 645 & 419 & 1 & & 127 & 67 & 7 & 8 & 3 & 7 & 5298 \\
\hline 0 & 41 & 1 & 0 & 7 & 0 & 4 & 3 & 0 & 52 & & 0 & 559 & 291 & 0 & 3 & 2369 \\
\hline 0 & 0 & 0 & 0 & 0 & 0 & 0 & 0 & 0 & 0 & 0 & & 0 & 0 & 0 & 0 & 69 \\
\hline 2 & 0 & 148 & 55 & 6 & 0 & 10 & 147 & 0 & 90 & 8 & 0 & t & 0 & 0 & 0 & 1460 \\
\hline 24 & 3 & 19 & 295 & 26 & 1 & 4 & 34 & 2 & 8 & 4 & 0 & 0 & & 0 & 0 & 1512 \\
\hline 0 & 2 & 2 & 15 & 5 & 0 & 4 & 57 & 0 & 0 & 3 & 0 & 0 & 0 & & 3 & 223 \\
\hline 0 & $\theta$ & 6 & 16 & 18 & 3 & 64 & 66 & 7 & 0 & 0 & 1 & 0 & 0 & 0 & & 956 \\
\hline
\end{tabular}

$\begin{array}{lllllllllllllllll}171 & 3641 & 1597 & 2493 & 5912 & 142 & 2936 & 7449 & 41 & 5433 & 2170 & 69 & 1436 & 1499 & 228 & 884 & 99829\end{array}$

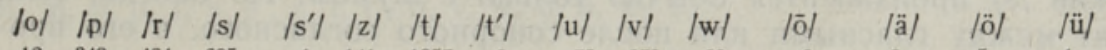

Таблица 4

\begin{tabular}{|c|c|c|c|c|c|c|c|c|c|c|c|c|c|c|c|}
\hline 12 & 349 & 484 & 605 & 1 & 141 & 1357 & 0 & 73 & 879 & 29 & 0 & 0 & 5 & 1 & $|a|$ \\
\hline 36 & 0 & 11 & 1 & 0 & 2 & 3 & 0 & 179 & 0 & 0 & 10 & 66 & 4 & 7 & $/ b /$ \\
\hline 98 & 0 & 136 & 2 & 0 & 0 & 0 & 0 & 407 & 1 & 0 & 7 & 12 & 27 & 103 & $|d|$ \\
\hline 10 & 10 & 0 & 0 & 0 & 0 & 0 & 0 & 0 & 0 & 0 & 0 & 0 & 0 & 0 & $\left|\mathrm{~d}^{\prime}\right|$ \\
\hline 36 & 357 & 261 & 1478 & 1 & 918 & 1538 & 1 & 35 & 193 & 34 & 46 & 110 & 45 & 0 & $|e|$ \\
\hline 3 & 1 & 4 & 5 & 0 & 10 & 10 & 0 & 6 & 1 & 0 & 0 & 0 & 1 & 1 & [f] \\
\hline 95 & 0 & 83 & 25 & 0 & 6 & 1 & 0 & 278 & 0 & 0 & 0 & 122 & 6 & 8 & $|g|$ \\
\hline 248 & 0 & 0 & 0 & 0 & 0 & 0 & 0 & 136 & 0 & 0 & 82 & 205 & 0 & 138 & {$[\mathrm{~h} /$} \\
\hline 57 & 256 & 524 & 859 & 29 & 417 & 491 & 9 & 402 & 292 & 0 & 600 & 304 & 7 & 4 & /i/ \\
\hline 31 & 1 & 139 & 54 & 69 & 27 & 69 & 0 & 61 & 3 & 0 & 11 & 0 & 1 & 6 & $\mid \mathrm{j} /$ \\
\hline 76 & 5 & 31 & 285 & 16 & 98 & 238 & 0 & 202 & 11 & 0 & 3 & 57 & 8 & 110 & {$[\mathrm{k} /$} \\
\hline 929 & 33 & 5 & 34 & 1 & 44 & 236 & 0 & 587 & 5 & 0 & 43 & 62 & 14 & 179 & 11/ \\
\hline 5 & 0 & 0 & 0 & 0 & 0 & 0 & 0 & 10 & 0 & 0 & 1 & 88 & 1 & 27 & $\left|I^{\prime}\right|$ \\
\hline 232 & 4 & 62 & 65 & 0 & 93 & 185 & 1 & 197 & 2 & 0 & 19 & 2 & 6 & 155 & $/ \mathrm{m} /$ \\
\hline 988 & 1 & 20 & 46 & 0 & 66 & 136 & 0 & 328 & 2 & 0 & 104 & 49 & 1 & 9 & $/ \mathrm{n} /$ \\
\hline 6 & 0 & 0 & 0 & 0 & 0 & 1 & 0 & 34 & 0 & 0 & 0 & 0 & 0 & 0 & $\left|\mathrm{n}^{\prime}\right|$ \\
\hline \multirow[t]{2}{*}{10} & 0 & 0 & 0 & 0 & 0 & 0 & 0 & 5 & 0 & 0 & 2 & 24 & 0 & 0 & $|\boldsymbol{\eta}|$ \\
\hline & 149 & 154 & 179 & 2 & 146 & 282 & 1 & 44 & 41 & 0 & 0 & 3 & 2 & 0 & lo/ \\
\hline 46 & & 10 & 152 & 0 & 50 & 95 & 00 & 76 & 1 & 0 & 148 & 19 & 2 & 5 & $\mid \mathrm{p} /$ \\
\hline \multirow[t]{2}{*}{323} & 116 & & 7 & 0 & 23 & 177 & 0 & 252 & 0 & 0 & 55 & 295 & 15 & 16 & $|r|$ \\
\hline & & 32 & & 0 & 137 & 600 & 17 & 406 & 7 & 0 & 6 & 26 & 5 & 18 & $|\mathrm{~s}|$ \\
\hline 1 & 0 & 0 & 0 & & 0 & 0 & 0 & 14 & 0 & 0 & 0 & 1 & 0 & 3 & $\left|\mathrm{~s}^{\prime}\right|$ \\
\hline 83 & 0 & 7 & 0 & 0 & & 0 & 0 & 569 & 4 & 0 & 10 & 4 & 3 & 64 & $|z|$ \\
\hline 119 & 15 & 120 & 1564 & 15 & 78 & & 1 & 412 & 3 & 0 & 147 & 34 & 57 & 66 & $|t|$ \\
\hline 17 & 0 & 0 & 0 & 0 & 0 & 0 & & 1 & 0 & 0 & 0 & 2 & 0 & 7 & $\left|t^{\prime}\right|$ \\
\hline 5 & 150 & 109 & 395 & 0 & 126 & 979 & 0 & & 52 & 0 & 89 & 0 & 0 & 0 & $|\mathrm{u}|$ \\
\hline 38 & 1 & 122 & 98 & 1 & 73 & 126 & 2 & 126 & & 0 & 8 & 4 & 3 & 0 & $|v|$ \\
\hline 0 & 0 & 0 & 0 & 0 & 0 & 0 & 0 & 67 & 0 & & 0 & 0 & 0 & 0 & $|\mathbf{w}|$ \\
\hline 1 & 75 & 13 & 111 & 0 & 7 & 80 & 0 & 7 & 559 & 0 & & 0 & 0 & 0 & $|\bar{\sigma}|$ \\
\hline 0 & 141 & 46 & 4 & 0 & 11 & 216 & 0 & 8 & 291 & 0 & 0 & & 0 & 0 & |ä| \\
\hline 0 & 10 & 0 & 9 & 0 & 1 & 137 & 0 & 3 & 0 & 0 & 0 & 0 & & 0 & /ö/ \\
\hline 3 & 13 & 16 & 50 & 1 & 10 & 70 & 1 & 7 & 3 & 0 & 0 & 0 & 3 & & (u) \\
\hline
\end{tabular}




\begin{tabular}{|c|c|c|c|c|c|c|c|c|c|c|c|c|c|c|c|}
\hline & $\mid \mathrm{a} /$ & /b/ & $/ \mathrm{d} /$ & $\left|\mathrm{d}^{\prime}\right|$ & $\mid \mathrm{e} /$ & $\mid \mathrm{f} /$ & $|\mathrm{g}|$ & $/ \mathrm{h} /$ & /i/ & $\mid \mathrm{j} /$ & $|\mathrm{k}|$ & III & $\left|I^{\prime}\right|$ & $/ \mathrm{m} /$ & $/ \mathrm{n}$ \\
\hline$|\mathrm{a}|$ & & 340 & 692 & 3 & 364 & 13 & 414 & 464 & 337 & 360 & 822 & 1066 & 127 & 694 & 747 \\
\hline$|\mathrm{b}|$ & 176 & & 1 & 0 & 111 & 1 & 1 & 0 & 113 & 16 & 56 & 40 & 0 & 34 & \\
\hline & 1102 & 0 & & 0 & 485 & 2 & 2 & 0 & 329 & 50 & 168 & 120 & 0 & 137 & \\
\hline$\left|\mathrm{d}^{\prime}\right|$ & 0 & 0 & 0 & & 0 & 0 & 0 & 0 & 13 & 0 & 0 & 0 & 0 & 0 & \\
\hline lel & 688 & 156 & 628 & 0 & & 18 & 486 & 164 & 994 & 140 & 764 & 1493 & 42 & 707 & \\
\hline$|\mathrm{f}|$ & 7 & n & 0 & 0 & 12 & & 0 & 0 & 38 & 0 & 0 & 1 & 0 & 0 & \\
\hline$|g|$ & 717 & 0 & 1 & 0 & 247 & 0 & & 0 & 553 & 3 & 6 & 11 & 0 & 12 & \\
\hline h/ & 242 & 0 & 0 & 0 & 455 & 0 & 0 & & 74 & 32 & 89 & 1 & 0 & 14 & \\
\hline |i/] & 134 & 154 & 1007 & 0 & 128 & 6 & 244 & 96 & & 318 & 932 & 585 & 3 & 666 & 114 \\
\hline$|\mathrm{j}|$ & 789 & 0 & . & 0 & 222 & 0 & 0 & 0 & 0 & & 0 & 0 & 0 & 0 & \\
\hline $\mid \mathrm{k} /$ & 854 & 4 & 0 & 0 & 334 & 0 & 0 & 1 & 318 & 8 & & 52 & 0 & 22 & \\
\hline III & 525 & 9 & 177 & 0 & 1747 & 2 & 87 & 6 & 976 & 52 & 110 & & 0 & 208 & 10 \\
\hline$I^{\prime \prime} I$ & 1 & 3 & 8 & 0 & 2 & 0 & 13 & 0 & 20 & 210 & 5 & 1 & & 21 & \\
\hline $\mid \mathrm{m} /$ & 1009 & 61 & 0 & 0 & 894 & 1 & 12 & 1 & 835 & 7 & 18 & 12 & 0 & & \\
\hline$/ \mathrm{n} /$ & 654 & 2 & 602 & 0 & 908 & 15 & 63 & 0 & 657 & 54 & 150 & 31 & 0 & 95 & \\
\hline$\left|\mathrm{n}^{\prime}\right|$ & 0 & 0 & 57 & 0 & 0 & 0 & 0 & 0 & 40 & 1 & 0 & 0 & 0 & 0 & \\
\hline$|\eta|$ & 0 & 0 & 0 & 0 & 0 & 0 & 161 & 0 & 0 & 0 & 10 & 0 & 0 & 0 & \\
\hline 101 & 13 & 33 & 99 & 10 & 36 & 3 & 94 & 277 & 61 & 32 & 75 & 903 & 5 & 213 & 10 \\
\hline$|p|$ & 315 & 0 & 0 & 0 & 328 & 1 & 0 & 0 & 250 & 1 & 5 & 32 & 0 & 4 & \\
\hline$|r|$ & 517 & 13 & 145 & 0 & 269 & 4 & 83 & 0 & 536 & 139 & 32 & 5 & 0 & 62 & \\
\hline$|s|$ & 581 & 1 & 2 & 0 & 1269 & 5 & 25 & 0 & 649 & 54 & 285 & 34 & 0 & 65 & \\
\hline$\left|\mathbf{s}^{\prime}\right|$ & 1 & 0 & 0 & 0 & 1 & 0 & 0 & 0 & 32 & 69 & 16 & 1 & 0 & 0 & \\
\hline$|z|$ & 152 & 2 & 0 & 0 & 936 & 10 & 6 & 0 & 501 & 27 & 98 & 44 & 0 & 93 & \\
\hline /t/ & 1408 & 3 & 0 & 0 & 1536 & 10 & 1 & 0 & 534 & 69 & 238 & 236 & 0 & 186 & 13 \\
\hline$\left|t^{\prime}\right|$ & 0 & 0 & 0 & 0 & 1 & 0 & 0 & 0 & 9 & 0 & 4 & 0 & 0 & 1 & \\
\hline$|u|$ & 73 & 199 & 617 & 0 & 35 & 6 & 278 & 134 & 406 & 61 & 204 & 612 & 11 & 195 & 332 \\
\hline$|\mathrm{v}|$ & 830 & 0 & 1 & 0 & 187 & 1 & 0 & 0 & 283 & 3 & 11 & 5 & 0 & 2 & \\
\hline$|\mathbf{w}|$ & 34 & 0 & 0 & 0 & 35 & 0 & 0 & 0 & 0 & 0 & 0 & 0 & 0 & 0 & \\
\hline$|\overline{\mid}|$ & 0 & 10 & 0 & 0 & 46 & 0 & 0 & 79 & 660 & 11 & 3 & 42 & 1 & 18 & 10 \\
\hline |ä| & 0 & 72 & 12 & 0 & 113 & 0 & 122 & 208 & 306 & 0 & 57 & 61 & 88 & 2 & \\
\hline /के/ & 5 & 5 & 30 & 0 & 43 & 1 & 6 & 0 & 7 & 1 & 8 & 16 & 1 & 6 & \\
\hline /ü/ & 1 & 7 & 109 & 0 & 0 & 1 & 8 & 113 & 4 & 6 & 90 & 187 & 26 & 155 & \\
\hline
\end{tabular}

Эстонский /s/ произносится обычно только с шумом. Но иногда, если он стоит между гласными или после сонорного согласного, в его произношении принимают участие и голосовые связки, в результате чего шум сопровождается тональным компонентом. В этих случаях /s/ воспринимается как полузвонкий и обозначается здесь как $/ \mathrm{z} /$.

В эстонском языке $/ \mathrm{n} /$ может быть обычным, палатализованным и назальным. Эти звуки выполняют смыслоразличительную функцию. Обычный встречается в любом фонетическом окружении, а назальный лишь в комбинациях $/ \mathrm{ng} /$ и $/ \mathrm{nk} /$. Он отмечен здесь как $/ \eta /$. Гласный $/ \mathrm{i} /$ и полугласный /j/ в фонетическом отношении почти одинаковы, только /i/ произносится сильнее, яснее и имеет все степени долготы. /j/ встречается только в начальной позиции слога и слова. Здесь они рассматриваются как два разных звука.

В данной работе долгота звуков, которая также изменяет смысл слов, во внимание не принимается.

В эстонском языке $b, d$ и $g$ используются для индикации кратких форм $/ \mathrm{p} /, / \mathrm{t} /$ и $/ \mathrm{k} /$. Однако в некоторых случаях /b/, /d/ и /g/ отличаются от $/ \mathrm{p} /, \mathrm{t} /$ и $/ \mathrm{k} /$ не только уровнем интенсивности, но и спектром и способом произношения. Глухие $/ \mathrm{p} /$, / $/$ / и $/ \mathrm{k} /$ произносятся путем кратковременного полного смыкания голосового тракта и затем быстрого раскрытия преграды. В зависимости от манеры раскрытия рта произносятся $/ \mathrm{p} /, / \mathrm{t} /$ или $/ \mathrm{k} /$. Если /b/, /d/ или /g/ стоят перед гласной, голосовые связки дают короткие импульсы в пределах основного тона, которые быстро исчезают до окончания произношения. Поэтому /b/, /d/ и /g/ приняты здесь за разные звукотипы $/ \mathrm{p} /, / \mathrm{t} /$ и $/ \mathrm{k} /$ и условно названы полузвонкими взрывными. Итак, в эстонской устной речи нами выде- 
Таблица 5

\begin{tabular}{|c|c|c|c|c|c|c|c|c|c|c|c|c|c|c|c|c|}
\hline$/ n^{\prime} \mid$ & $/ \eta /$ & $10 \mid$ & $|\mathrm{p}|$ & $|\mathrm{r}|$ & $|\mathrm{s}|$ & $\left|s^{\prime}\right|$ & $|z|$ & $|t|$ & $\left|t^{\prime}\right|$ & $|\mathrm{u}|$ & $|\mathrm{v}|$ & $|\mathrm{w}|$ & $|\overline{0}|$ & |ä/ & | & /ü/ \\
\hline 20 & 17 & 252 & 284 & 457 & 982 & 112 & 521 & 1154 & 7 & 146 & 429 & 0 & 33 & 14 & 8 & 91 \\
\hline 0 & 0 & 83 & 22 & 27 & 74 & 0 & 0 & 39 & 0 & 26 & 43 & 0 & 4 & 37 & 3 & 18 \\
\hline 0 & 0 & 154 & 85 & 37 & 176 & 0 & 6 & 112 & 0 & 347 & 132 & 0 & 15 & 14 & 0 & 34 \\
\hline 0 & 0 & 0 & 0 & 0 & 0 & 0 & 0 & 0 & 0 & 0 & 0 & 0 & 0 & 0 & 0 & 0 \\
\hline 65 & 1 & 291 & 284 & 412 & 889 & 5 & 460 & 743 & 7 & 25 & 430 & 1 & 39 & 20 & 5 & 49 \\
\hline 0 & 0 & 27 & 1 & 0 & 0 & 0 & 0 & 0 & 0 & 4 & 0 & 0 & 0 & 1 & 1 & 9 \\
\hline 0 & 0 & 10 & 3 & 36 & 15 & 0 & 0 & 4 & 0 & 540 & 4 & 0 & 1 & 0 & 0 & 102 \\
\hline 0 & 0 & 12 & 2 & 0 & 14 & 0 & 0 & 339 & 0 & 61 & 54 & 0 & 0 & 0 & 0 & 0 \\
\hline 6 & 112 & 355 & 164 & 139 & 1206 & 3 & 1062 & 872 & 0 & 30 & 253 & 0 & 26 & 16 & 20 & 36 \\
\hline 0 & 0 & 39 & 0 & 0 & 0 & 0 & 0 & 0 & 0 & 494 & 0 & 0 & 38 & 145 & 0 & 1 \\
\hline 0 & 0 & 701 & 9 & 41 & 804 & 0 & 0 & 96 & 0 & 564 & 21 & 0 & 165 & 104 & 1 & 164 \\
\hline 0 & 0 & 224 & 53 & 11 & 71 & 0 & 24 & 562 & 0 & 234 & 57 & 0 & 45 & 136 & 5 & 42 \\
\hline 0 & 0 & 1 & 0 & 0 & 1 & 0 & 0 & 15 & 0 & 1 & 1 & 0 & 0 & 0 & 0 & 1 \\
\hline 0 & 0 & 102 & 27 & 1 & 19 & 0 & 8 & 20 & 0 & 258 & 20 & 0 & 121 & 76 & 12 & 17 \\
\hline 0 & 0 & 123 & 55 & 17 & 230 & 1 & 6 & 200 & 0 & 408 & 112 & 1 & 49 & 149 & 6 & 100 \\
\hline & 0 & 0 & 0 & 0 & 10 & 0 & 0 & 22 & 0 & 0 & 0 & 0 & 0 & 0 & 0 & 0 \\
\hline 0 & & 0 & 0 & 0 & 0 & 0 & 0 & 0 & 0 & 0 & 0 & 0 & 0 & 0 & 0 & 0 \\
\hline 6 & 9 & & 43 & 323 & 95 & 0 & 85 & 114 & 15 & 6 & 39 & 0 & 1 & 0 & 0 & 3 \\
\hline 0 & 0 & 144 & & 103 & 34 & 0 & 0 & 15 & 0 & 142 & 1 & 0 & 63 & 137 & 10 & 9 \\
\hline 0 & 0 & 151 & 10 & & 33 & 0 & 7 & 123 & 0 & 115 & 128 & 0 & 12 & 38 & 0 & 0 \\
\hline 0 & 0 & 178 & 151 & 7 & & 0 & 0 & 1760 & 0 & 371 & 98 & 0 & 106 & 2 & 8 & 47 \\
\hline 0 & 0 & 2 & 0 & 0 & 0 & & 0 & 16 & 0 & 0 & 1 & 0 & 0 & 0 & 0 & 0 \\
\hline 0 & 0 & 146 & 50 & 23 & 137 & 0 & & 78 & 0 & 133 & 73 & 0 & 7 & 11 & 1 & 10 \\
\hline 1 & 0 & 274 & 95 & 174 & 607 & 0 & 0 & & 0 & 980 & 126 & 0 & 72 & 167 & 138 & 69 \\
\hline 0 & 0 & 1 & 0 & 0 & 17 & 0 & 0 & 0 & & 0 & 2 & 0 & 0 & 0 & 0 & 1 \\
\hline 34 & 5 & 44 & 76 & 252 & 413 & 14 & 644 & 418 & 1 & & 126 & 64 & 7 & 8 & 3 & 7 \\
\hline 0 & 0 & 37 & 1 & 0 & 7 & 0 & 4 & 3 & 0 & 52 & & 0 & 453 & 260 & 0 & 3 \\
\hline 0 & 0 & 0 & 0 & 0 & 0 & 0 & 0 & 0 & 0 & 0 & 0 & & 0 & 0 & 0 & 0 \\
\hline 0 & 0 & 0 & 138 & 55 & 6 & 0 & 10 & 147 & 0 & 90 & 8 & 0 & & 0 & 0 & 0 \\
\hline 0 & 0 & 3 & 19 & 293 & 22 & 1 & 4 & 34 & 2 & 0 & 4 & 0 & 0 & & 0 & 0 \\
\hline 0 & 0 & 2 & 2 & 15 & 5 & 0 & 4 & 57 & 0 & 0 & 3 & 0 & 0 & 0 & & 3 \\
\hline 0 & 0 & 0 & 6 & 15 & 18 & 3 & 64 & 55 & 7 & 0 & 0 & 0 & 0 & 0 & 0 & \\
\hline
\end{tabular}

лены следующие 32 звукотипа: /a, b, d, d', e, f, g, h, i, j, k, l, l', m, n, $\mathrm{n}^{\prime}, \eta, \mathrm{o}, \mathrm{p}, \mathrm{r}, \mathrm{s}, \mathrm{s}^{\prime}, \mathrm{z}, \mathrm{t}, \mathrm{t}^{\prime}, \mathrm{u}, \mathrm{v}, \mathrm{w}, \tilde{\mathrm{o}}, \ddot{\mathrm{a}}, \ddot{\mathrm{o}}, \ddot{\mathrm{u}} /$.

\section{Таблицы}

В табл. 1 приведены перечисленные выше звукотипы и слова, их характеризующие. Из 32 звукотипов огносительная частота появления девяти гласных составляет $45,66 \%$, а из всех гласных наиболее употребительны /a, e, i/ - 33,02\%, причем /a/ встречается в 53 раза чаще, чем /ӧ/. Гласный /i/ появляется в 4 раза чаще полугласного /j/ (на письме в 6 раз чаще). Обращает на себя внимание тот факт, что половина встречаемости всех гласных приходится на гласные /a, е/. Частота появления $/ \mathrm{k}, \mathrm{l}, \mathrm{n}, \mathrm{s}, \mathrm{t} /$ составляет больше половины встречаемости всех остальных согласных, вместе взятых. Среди перечисленных пяти звукотипов наиболее распространен /t/ (на письме $s$ ). Из взрывных согласных /t, t'/ появляются примерно в 5 раз чаще, чем $/ \mathrm{p} /$, и в 2 раза чаще, чем $/ \mathrm{k} /$. В табл. 2 звукотипы сгруппированы согласно их артикуляции и часто́те появления в речи.

В табл. 3 приведены данные о частотности двухзвуковых сочетаний (диграмм). По вертикали выписаны первые и по горизонтали вторые звуки диграмм. Интересно отметить следующее. В отдельных словах печатного текста нами было выявлено 25 разных дифтонгов, причем на первом месте могут стоять все гласные, а на втором - гласные $a, e, i, o$. В устной же речи количество различных гласных диграмм в синтагмах увеличивается до 53, и в первой позиции могут находиться все гласные. Теоретически из девяти гласных можно образовать 72 сочетания. Сле- 
довательно, в синтагмах встрегилось из них 73,61\%. В нашем материале зафиксировано 586 разных диграмм из возможных 992, или $59,07 \%$. Из всех 99829 диграмм более 10 раз встретилась 99241 диграмма в 416 комбинациях, более 5 раз 516 диграмм в 106 комбинациях и по одному разу 72 диграммы. Гять наиболее употребительных диграмм: /st/ - 1763, /le/ - 1761, /te/ - 1602, /se/ - 1583, /el/ - 1504 (на письме из 94010 диад, образованных из 102307 букв, наиболее используемыми были следующие: se - 2624, is - 2011, st - 1751, te - 1708, es - 1566).

Теоретически из 32 звукотипов можно создать 29760 трехзвуковых сочетаний (триграмм) без повторения рядом одинаковых звуков. Практически же удалось выявить только 3026 таких сочетаний, или $10,16 \%$. Перечислим пять с высокой частотностью: /ele/ - 526, /ist/ - 489, /sel/ - 464, lest/ - 400, /ole/ - 398 (на пнсьме: ste - 266, sel - 230, use -224 , est - 216 и mis - 197). По одному разу встретилось 950 триграмм. В табл. 4 приведены правые и в табл. 5 левые соседи центральных звуков. Последние выписаны на горизонтальной линии таблиц.

Относительная частота появления звуков в процентном выражении вычисляется по формуле $z_{i}=x_{i} / n \cdot 100$, где $x-$ частотность данного звука $i$ среди всех звуков $n$, вероятность появления $p_{i}$ которого неизвестна. Располагая большим количеством $n$, можно предполагать, что $p_{i} \approx z_{i}$. Поскольку $z_{i}$ является лишь одним значением случайной величины, желательно знать ее доверительные границы. При $99 \%$-ной доверительной вероятности изменчивость переменной величины выражается формулой

$$
p_{1,2}^{\prime}=x / n \pm 2,576\left[x(n-x) / n^{3}\right]^{1 / 2} .
$$

Результаты расчета приведены в табл. 1. Например, для /а/, абсолютная частотность которого 12304 из 105972 звуков и относительная частотность $11,61 \%$, доверительные границы равны $11,36-11,87 \%$. Следовательно, с $99 \%$-ной вероятностью можно прогнозировать, что в эстонской устной речи /a/ встретится от 11360 до 11870 раз на каждые 100000 звуков.

\section{Заключение}

Проведен статистический анализ частотности появления отдельных звуков, двух-и трехзвуковых сочетаний в произвольно выбранных отрезках эстонской устной речи. Установлено, что наиболее употребительными являются звук/a/, диграмма/st/ и триграмма/ele/ (в печатном тексте буква $a$, диада se и триада ste). Слово в устной речи состоит в среднем из 5,4 звука и синтагма из 22,5 звука (в печатном тексте эти данные равны соответственно 7 и 35 буквам). Синтагма содержит сочетания звуков, отсутствующие в отдельных словах. Результаты исследования могут быть использованы прн разработке правил управления синтезатором речевых сигналов.

\section{ЛИТЕРАТУ РА}

1. Кюн н а п Э., Изв. АН ЭССР, Физ. Матем., 26, № 4, 424-430 (1977). 


\section{E. KONNAP}

\section{EESTI KONEKEELE STATISTIKA}

Artiklis on analüüsitud eesti kõnekeelt ja toodud andmeid häälikute ning nendest koosnevate digrammide ja trigrammide esinemissageduse kohta. Kokku on uuritud 105942 häälikut, mis moodustavad 19620 sõna ja 4923 süntagmat.

\section{E. KUNNAP}

\section{ON THE STATISTICS OF SPOKEN ESTONIAN}

Different combinations of sounds are used to synthesize fluent speech. Every sound in a syllable has some influence upon the final state of the preceeding sound as well as on the following sound. Therefore, in order to investigate the rules of the control of a synthesizer of human speech, it is necessary to have data about the probability of the appearance of the sounds in fluent speech, to find out which combinations are more frequent and which combinations do not exist at all.

Conversation differs from written text by both the duration of a phrase and the composition of words in the phrase. Very often in conversation the endings of words are pronounced with phonetic mistakes or are not pronounced at all, sometimes some parasite words which have no sense and do not exist in written text, are added. The other aim of this work is to obtain the statistical data on spoken Estonian and the differences between the latter and the written text in both the frequency of occurrence of sounds and the composition of digrams and trigrams. The analysis was made by syntagmas, i.e. from pause to pause in real speech. The material is based on random fragments from the speech between two or more persons, from the conversation held at meetings, in shops, radio and TV announcements. All in all 105942 sounds were investigated forming 19620 words and 4923 syntagmas. In Tables $3-5$ the frequency of occurrence of sounds as well as digrams and trigrams are shown. 